\title{
Construcción subjetiva del territorio: Experiencias del habitar la provincia del Bio Bio, Chile
}

Rodolfo E. Mardones. Universidad Austral de Chile Jorge Ulloa. Universidad Santo Tomás

\section{Resumen}

El objetivo de este artículo fue "comprender la construcción subjetiva del territorio de habitantes de la ciudad, el campo y el territorio indígena de la provincia de Bio Bio, Chile". En particular, se caracteriza y compara el relato de los participantes en torno a la construcción del territorio. Se investiga desde una perspectiva cualitativa, realizando una recolección de datos por medio de la combinación de dos estrategias: la deriva y la entrevista en profundidad. Los datos fueron analizados por medio del análisis temático de contenido. Los resultados se presentan en función de las temáticas teóricas estudiadas en cada territorio. Se concluye que la construcción subjetiva del territorio se ve marcada por la relación entre la materialidad y lo simbólico, la relación con otros y la experiencia emocional con los lugares.

Palabras clave: construcción subjetiva; territorio; apropiación del espacio; relaciones comunitarias.

\section{Resumo}

Construção subjetiva do território: Experiências del habitar a província de Bio Bio, Chile. O objetivo deste artigo foi "compreender a construção subjetiva do território de moradores da cidade, o campo e o território indígena da província de Bio Bio, Chile". Em particular, caracteriza-se e compararam a discursos dos participantes sobre a construção do território. Ele é investigado a partir de uma perspectiva qualitativa, realizando uma coleta de dados através da combinação de duas estratégias: deriva e entrevistas em profundidade, que foram analisadas pela análise temático de conteúdo. Os resultados são apresentados em termos de conteúdos teóricos estudados em cada território. Conclui-se que a construção subjetiva do território é marcada pela relação entre o material e o simbólico, relação com os outros, e experiência emocional com lugares.

Palavras-chave: construção subjetiva; território; apropriação do espaço; relações com a comunidade.

\begin{abstract}
Subjective construction of the territory: Experiences of dwell the province of Bio Bio, Chile. The objective of this article was "to understand the subjective construction of the territory of inhabitants of the city, the countryside and the indigenous territory of the province of Bio Bio, Chile". In particular, the story of the participants about the construction of the territory is characterized and compared. It is investigated from a qualitative perspective, making a data collection through the combination of two strategies: the drift and the in-depth interview, which were analyzed through thematic analysis of content. The results are presented according to the theoretical themes studied in each territory. It is concluded that the subjective construction of the territory is marked by the relationship between materiality and symbolism, relationship with others and emotional experience with places.
\end{abstract} Keywords: subjective construction; territory; appropriation of space; community relations. 
El concepto de territorio ha sido estudiado desde perspectivas variadas y en vinculación a tópicos diversos que lo configuran material y simbólicamente (Mardones, 2016). Desde la psicología, el estudio de la relación entre los sujetos, sus comunidades y el medio geográfico se ha fundamentado a partir de conceptualizaciones como la apropiación del espacio, apego al lugar, identidad de lugar, arraigo y relaciones comunitarias, entre otras. Estos conceptos permiten problematizar y complejizar las formas en que el territorio es, por ejemplo, vivido, significado, apropiado o narrado. Lo cual permite abordar el territorio en su amplia complejidad y estructura geográfica, tomando en consideración lo urbano y lo rural, entendidos como territorios constituidos en interacciones complejas por sujetos, objetos y discursos que se despliegan en el espacio. Es en este punto donde este artículo pretende contribuir, complejizando la construcción del territorio desde la perspectiva de sus habitantes, relevando el rol del sujeto.

\section{Apropiación del Espacio}

Uno de los principales conceptos utilizados para la descripción de la relación persona-ambiente en la psicología ambiental y otras ciencias sociales en deslinde, es la apropiación del espacio, entendido como un concepto temporal y dinámico de interacción entre la persona y su medio (Korosec-Serfaty, 1976). Este concepto se refiere a los procesos mediante los cuales los individuos se apropian del contexto espacial a través de la interacción sociocultural, gracias a la cual las experiencias de otros son internalizadas como una realidad propia (Vidal \& Pol, 2005; Vidal, Pol, Guàrdia, \& Peró, 2004), nos condiciona, nos fija, nos sujeta y define por su configuración (Villela, 1976). La apropiación no se relaciona con el espacio geográfico exclusivamente, sino también con los significados sociales adquiridos durante una continua interacción (Vidal et al., 2004); existe una relación simbólica y transformadora (Pol, 1996). Parte de la literatura clásica en psicología social ha advertido que, mediante la acción, los sujetos transforman el espacio a través de los procesos de interacción con otros; mientras que, por medio de la identificación simbólica, la persona y el grupo se reconocen en el entorno, mediante procesos de categorización del yo (Turner, 1990).

Se considera la apropiación de espacios en una relación estrecha con el apego al lugar y la identidad del lugar (Vidal, Berroeta, Di Masso, Valera, \& Peró, 2013; Vidal \& Pol, 2005; Vidal et al., 2004), como expresiones de las relaciones socioespaciales constituidas por aspectos cognitivos, emocionales y conductuales. El concepto de apego al lugar da cuenta principalmente de los vínculos emocionales con los lugares (Altman \& Low, 1992; Hidalgo, 1998; Lewicka, 2010; Tuan, 2001); tal como puntualizan Hidalgo y Hernández (2001): "como un enlace o vínculo afectivo entre las personas y lugares específicos" (p. 274). Giuliani (2003) establece que esta definición ayuda a diferenciar el apego al lugar de otros conceptos que tienden a orientarse hacia lo cognitivo, conductual o simbólico. Otro elemento a dilucidar es que el tipo de afecto que se tiene con el lugar es, principalmente, de carácter positivo (Altman \& Low, 1992; Giuliani \& Feldman, 1993). Sin embargo, también puede ser ambivalente e incluso negativo. En resumen, una definición más concreta y consensuada considera el apego al lugar como un vínculo afectivo y específico de las personas hacia determinados lugares con los que se relacionan (Hidalgo, 1998).

Dentro de las diversas aplicaciones y usos del concepto de apego al lugar, ha tenido mayor presencia en investigaciones de tipo cuantitativo, utilizándose escalas y modelos, como el propuesto por Scannell y Gifford (2010), o diversas escalas de actitudes para medir el constructo de apego, como los propuestos por Amérigo (1990), Harris, Brown y Werner (1996), Hidalgo y Hernández (2001), Jorgensen y Stedman (2001), Vidal, Valera y Peró (2010) y Qinjiu y Maliki (2013), entre otros.

En los últimos años, diversos estudios dan cuenta sobre la relación del concepto de apego al lugar con diversos procesos de mejora de la calidad de vida en el barrio (Vidal et al, 2013; Vidal et al., 2010), destacando algunas variables relacionadas con el apego como: el tiempo de residencia, las expectativas de permanecer en el lugar actual, el número de viviendas anteriores, la relación del apego al lugar con el diseño de sus espacios, (Kamalipour, Yeganeh, \& Alalhesabi, 2012). Sumado a esto también se identifican estudios que analizan el proceso interpretativo relacional de distintos sujetos con sus espacios (Brown, Raymond, \& Corcoran, 2015; Casakin, Hernández, \& Ruiz, 2015; B. Hernández, Hidalgo, SalazarLaplace, \& Hess, 2007; Holton, 2015; Moghisi, Mokhtari, \& Heidari, 2015; Ujang \& Zakariya, 2015), los lugares de memoria (Fernández, 2007), así como los vínculos y significados locales (Mardones, 2013; 2014; 2016).

Por otro lado, otro concepto explicativo de las relaciones socioespaciales es la identidad de lugar, la cual tiene directa relación con las teorías del self en psicología social (Proshansky, 1978; Proshansky, Fabian, \& Kaminoff, 1983), en donde la identidad personal se construye en relación con el entorno físico (Berroeta, Ramoneda, Rodríguez, Di Masso, \& Vidal, 2015). En la 
misma línea, Devine-Wright y Clayton (2010) lo definen como "una forma de describir o conceptualizar al self, que puede incorporar funciones y atributos personales, la pertenencia a grupos o categorías sociales y conexiones con ubicaciones geográficas" (p. 267). Asimismo, Twigger-Ross y Uzzell (1996) sostienen que los lugares ayudan a reforzar aspectos de la identidad, y que esta está en la misma línea que la identidad social.

Recientes estudios empíricos dan cuenta de su utilización en conjunto con otros conceptos relacionados, como sentido de comunidad y apego al lugar (Berroeta et al., 2015; Casakin et al., 2015; B. Hernández et al., 2007; Qinjiu \& Maliki, 2013; Vidal et al., 2013; Vidal et al., 2010). Otros trabajos relevan la importancia de la identidad de lugar como indicador de satisfacción con el barrio o vecindario (Bernardo \& Palma-Olivera, 2016; Smith, Padgett, Choy-Brown, \& Henwood, 2015), extendiendo vínculos hacia una dimensión comunitaria de los procesos de apropiación de los espacios.

\section{Relaciones Comunitarias}

En las relaciones comunitarias, el concepto de comunidad constituye el eje central por su sentido polisémico (Berroeta \& Zambrano, 2012). Sin embargo, esta noción no ha logrado ser definida de manera unívoca, encontrándonos con un sinnúmero de definiciones en la literatura (e. g. Ander-Egg, 1980; Krause, 2001; Montero, 2004; Sánchez, 1996). Estas presentan énfasis distintos, sin embargo, existen nociones teóricas de interés compartido, en donde el rol del sujeto y sus capacidades de agencia se vuelven centrales. En este sentido, se define comunidad como una unidad simbólica relacionada con la vida en común (Hombrados-Medieta, 2010), cuya finalidad es responder a las demandas del entorno y satisfacer las necesidades compartidas (Krause, 2007; Martínez, 2006), en donde el territorio podría ocupar una dimensión central (Sánchez, 1996).

Por otro lado, así como en la literatura se presenta evidencia sobre la posibilidad de que los lugares generan identidad en los sujetos (Montenegro, Rodriguez, \& Pujol, 2014), estudios contemporáneos centran la noción de sujeto en la interacción que emerge del colectivo, dirigiendo la mirada a la dimensión social y no al espacio egocéntrico (Pantoja, 2011; Subercaseaux, et al., 2002). Por lo tanto, cuando se habla de identidad individual o colectiva, se hace referencia a procesos que permiten asumir que ésta persona, en determinado momento y contexto, es y tiene conciencia de sí (De la Torre, 2001; Gergen, 2007; Joutard, 1999). Por otro lado, la identidad no es un logro individual ni maduracional que emerge de adentro hacia afuera, sino que sería un proceso que surge de una dimensión narrativa que involucra relación, interacción y discurso dentro de un marco temporal (Giner, Lamo, \& Torres 1998). En este contexto, la identidad comunitaria se ha conceptualizado como una "noción otorgadora de sentido, que se expresa en acciones y verbalizaciones, que está cargada de afecto, que se construye históricamente y que se expresa en relaciones" (Montero, 2004, p. 106).

Un elemento conceptual complementario a lo presentado hasta ahora es el sentido de comunidad, entendido como proceso psicosocial, se define como un mecanismo por medio del cual el desarrollo comunitario estimula las oportunidades de pertenencia, influencia, necesidad de encuentro mutuo y de desarrollo de vínculos emocionales y apoyo entre los miembros de la comunidad (McMillan \& Chavis, 1986).

Mc Millan y Chavis (1986) plantean que el sentido de comunidad se compone de cuatro elementos: la membresía, influencia, integración y satisfacción de necesidades y conexión emocional compartida. Es considerado como el resultado de la intersección de distintas variables, cuya función es de carácter catalizador para desencadenar otros procesos comunitarios como el cambio social y la participación comunitaria. Constituye un constructo validado empíricamente en diversas áreas del conocimiento psicosocial, asociado con el prejuicio ético, la orientación política (Prezza, Zampatti, Pacilli, \& Paoliello, 2008) y la participación política. En el ámbito del quehacer comunitario y los estudios empíricos sobre el tema se presentan asociaciones con los significados que le otorgan los habitantes a sus comunidades, la aculturación de inmigrantes en países de acogida (Hombrados, Gómez-Jacinto, \& Domínguez-Fuentes, 2009), la satisfacción vital en vecindarios, el desarrollo de organizaciones comunitarias, relaciones con los aparatos de salud primaria (Farmer, Currie, Kenny, \& Munoz, 2015), aspectos de potenciación comunitaria en contextos urbanos y barriales (Zambrano, Bustamante, \& Garcia, 2009), así como también en su relación con el lugar y su incidencia en las acciones comunitarias desde una óptica colectiva (Ucar,2012) y, la relación intersubjetiva entre sujeto y espacio en contextos organizacionales institucionalizados (Iñiguez, 2005; Pantoja, 2011; Pérez, 2009). En este contexto, la participación toma un lugar central, en el sentido de reconocerse como parte de una comunidad con capacidades de acción y transformación. La participación es un concepto central en las relaciones comunitarias, se establece que para que esta sea tal, es imperativo que haya posibilidad de influencia recíproca entre los implicados y de incidencia 
en la toma de decisiones (Davis, 1982; Ferullo, 2006), mientras que, por otro lado, se señala que su generación es paulatina y por lo tanto existen diferentes tipos y grados (Ferullo, 2006) y se relaciona de forma directa con las conceptualizaciones del poder y la conversión (Krause y Jaramillo, 1998; Sánchez, 2013).

En relación con lo anterior, la participación tiene un carácter político, entendido desde la esfera de lo público, y se encuentra en estrecha relación con el compromiso. Su existencia en una localidad específica posibilita distintas formas de agenciamiento por parte de sujetos, minorías y procesos de participación local (Pick, Sirkin, Osorio, Xocolotzin, \& Givaudan., 2007).

Considerando las conceptualizaciones que fundamentan las nociones de apropiación del espacio (apego al lugar e identidad de lugar) y relaciones comunitarias (identidad comunitaria, sentido de comunidad y participación), este artículo se propone indagar de modo exploratorio y descriptivo ¿Cuáles son los discursos sobre las que se construye subjetivamente el territorio en las distintas escalas geográficas de la provincia del Bio Bio, Chile?

\section{Material y Método}

Este trabajó se realizó desde una perspectiva cualitativa, con el fin de abordar de una manera comprensiva el problema de investigación, a partir de los propios relatos de las personas, en sus palabras y en sus contextos (Taylor \& Bogdan, 1987). Desde esta comprensión se plantea como una investigación de carácter descriptiva (R. Hernández, Fernández, \& Baptista, 2006) realizada en base a un diseño flexible (Mardones, Ulloa, \& Salas, 2018) respecto a los contextos de investigación y el reconocimiento cultural de los/as participantes.

\section{Casos y Participantes}

Los casos de estudio se sitúan geográficamente en la provincia del Bío Bío, Chile, en distintas escalas geográficas, con el fin de realizar una aproximación a las distintas ópticas de construcción subjetiva del territorio. La provincia del Bio Bio se compone de 14 comunas, su capital es la ciudad de Los Ángeles y la población rural asciende a un $30 \%$ de la población total de la provincia. En su extensión se considera parte del territorio del pueblo Pehuenche.

Para este estudio, el territorio de la provincia se operacionaliza en los sectores: rural y urbano. La selección de los participantes se realizó por medio de un muestreo intencionado (Valles, 1999). Realizando una selección por conveniencia, en base a criterios previos, los cuales tenían la función de graficar los perfiles territoriales definidos. Se consideró a cuatro participantes del área urbana y cuatro del área rural. Es decir, cuatro casos urbanos para hacer la indagación en el centro de la ciudad, en la periferia urbana nivel socioeconómico bajo y en la periferia urbana nivel socioeconómico medio emergente. Esta distinción considera el grado de proximidad espacial o de aglomeración territorial de las familias pertenecientes a un mismo grupo social, en donde el nivel socioeconómico estaría asociado al fenómeno de segregación espacial presente en la ciudad (Azócar, Henríquez, Valenzuela, \& Romero, 2008). Mientras que se consideran cuatro casos rurales para hacer la indagación en el campo tradicional, la movilidad ciudad-campo y el territorio pehuenche.

Además, todos los participantes debieron cumplir los criterios de: ser mayor de edad, tener residencia de al menos 5 años en el territorio estudiado, la participación voluntaria.

\section{Técnicas de Producción de Datos}

Como método de investigación se utilizó la deriva. Esta proviene de estudios de la interfaz entre la psicología y la geografía y se entiende como el estudio de la afectividad y comportamiento de las personas en relación al medio geográfico (Pellicer, Nubiola, \& De Lacour, 2013). Para Debord (2006) las derivas se insertan dentro de las denominadas "psicogeografías", las que define como el estudio de los efectos específicos del entorno geográfico, conscientemente organizado o no, en las emociones y la conducta de los individuos. Por tanto, por medio de este método se hizo énfasis en la indagación de la afectividad involucrada en la significación del espacio y las relaciones comunitarias. En este sentido la metodología se orienta a conocer el territorio desde las personas que habitan en él cotidianamente, promoviendo una actitud consciente y reflexiva de las rutas y trayectos caminados (Montesinos, 2013). Es así, como la deriva permitió una lectura subjetiva de los distintos territorios. En donde el foco de exploración fueron las emociones en relación con el espacio habitado y con ello la cualificación del territorio.

Como técnicas de producción de datos se utilizó la entrevista en profundidad en torno a un registro fotográfico procedente de un recorrido por la ciudad entre el/la investigador/a y investigado/a, como procedimientos específicos: cada participante tuvo la posibilidad de capturar diez fotografías en una ruta definida por él/ella mismo/a en su espacio cotidiano. Por otro lado, para comprender los significados asociados al territorio, se realizaron entrevistas en profundidad con los participantes para abordar tópicos de interés (Valles, 1999), 
mediante un ejercicio conversacional no estructurado, abierto y dinámico basado en una pauta de temas. Estas se realizaron al terminar el recorrido y tuvieron una duración de una hora aproximadamente. En este caso, las fotografías definieron la pauta de temas de conversación y permitieron dar un hilo conductor al proceso de entrevista, siendo los elementos guías para reconocer las cualificaciones que cada participante hizo en torno al espacio habitado, y con ello el significado que atribuyen al territorio recorrido. Los aspectos éticos del estudio y las condiciones de colaboración de los participantes, así como el registro de los datos, fueron consensuados por medio de un consentimiento informado (Ávila, 2002).

\section{Análisis de Datos}

Se realizó un análisis temático de los discursos, facilitado por el uso del software de análisis de datos cualitativos Atlas.ti v7.2, este consistió en agrupar y organizar un conjunto de temas representativos de las entrevistas analizadas, configurando sistemas de discursos (Conde, 2009). Para facilitar esta tarea y la posterior exposición de los resultados, este artículo asume cinco ámbitos de análisis que funcionan como orientadoras del análisis abierto. Desde esta perspectiva, se asume que los discursos son producciones y prácticas sociales, actualizadas en la interacción social. Estos conforman un sistema estructurado, ordenado y jerarquizado con diversos grados de cristalización y de circulación social (Conde, 2009).

Tabla 1. Temáticas de Análisis

\begin{tabular}{ll}
\hline \multicolumn{1}{c}{ Dimensión estudiada } & \multicolumn{1}{c}{ Descripción } \\
\hline Representación del Territorio & $\begin{array}{l}\text { Elección visual de espacios y tematización conceptual del Territorio. } \\
\text { Aspectos materiales y cotidianos del Territorio. Se encuentran naturalizados y por lo tanto se aceptan como dados } \\
\text { de forma acrítica. }\end{array}$ \\
Territorios compartidos & $\begin{array}{l}\text { Reconoce a otras personas insertas en un todo del que se siente parte. Describe relaciones personales y/o sociales } \\
\text { inmersas en el Territorio, pueden ser pasadas, presentes o futuras. }\end{array}$ \\
$\begin{array}{l}\text { Reciprocidad con el Territorio } \\
\text { Problematización de la construcción } \\
\text { del Territorio }\end{array}$ & $\begin{array}{l}\text { Relación reciproca entre la persona y el espacio habitado, expone la emocionalidad que encierran los Territorios. } \\
\text { los espacios descritos. }\end{array}$ \\
\hline
\end{tabular}

Fuente: elaboración propia.

\section{Resultados}

La representación del territorio se construye en base a la selección de rutas y toma de fotografías. En el caso del territorio del centro urbano, tal elección se da por imágenes asociadas a la arquitectura, servicios, espacio público, vivienda y trabajo. En el caso de la periferia urbana, se comparten las temáticas de arquitectura, servicios y vivienda, sumando a estas la temática de "posesión de propiedades" como tema característico del territorio periurbano nivel socioeconómico medio emergente. En el caso del campo, el territorio se representa en base a imágenes asociadas a la naturaleza, trabajo, propiedad, espacio público y servicios. Mientras en el caso del territorio pehuenche, este se representa en torno a las nociones de naturaleza y vivienda; representaciones que difieren del sentido instrumental que se le otorga al territorio en el campo, ya que predomina la concepción cultural de espacios simbólicos en contraste a una concepción utilitaria o de propiedad privada.

Los aspectos materiales del territorio son definidos a partir de la descripción del espacio habitado.
En el caso del territorio del centro urbano, este es descrito en función del tamaño y ubicación de la vivienda, acceso a servicios, espacios públicos y patrimonio en función de sus condiciones de privilegio y seguridad. En el caso de la periferia urbana, los aspectos materiales definidos son: tamaño de la vivienda, exponiendo la tensión de un espacio reducido y uno amplio dependiendo del nivel socioeconómico, lo cual también se observa en la adquisición de bienes y en la descripción del entorno como caracterización de espacios de segregación residencial. En el caso del campo, el territorio se describe asociado a la propiedad de la tierra, al uso del terreno, la autoconstrucción, tamaño y comodidad de la vivienda (amplia y equipada para recibir a otras personas), en este caso, el territorio se define como útil y productivo. Por otro lado, el territorio pehuenche se describe materialmente en torno a infraestructuras que delimitan simbólicamente el territorio, elementos naturales y político-materiales como topónimos para la comunidad, diferenciándose en complejidad con la descripción instrumental de los otros territorios, atribuyendo un sentido simbólico protagónico. 
Tabla 2. Temas Discursivos en los Territorios

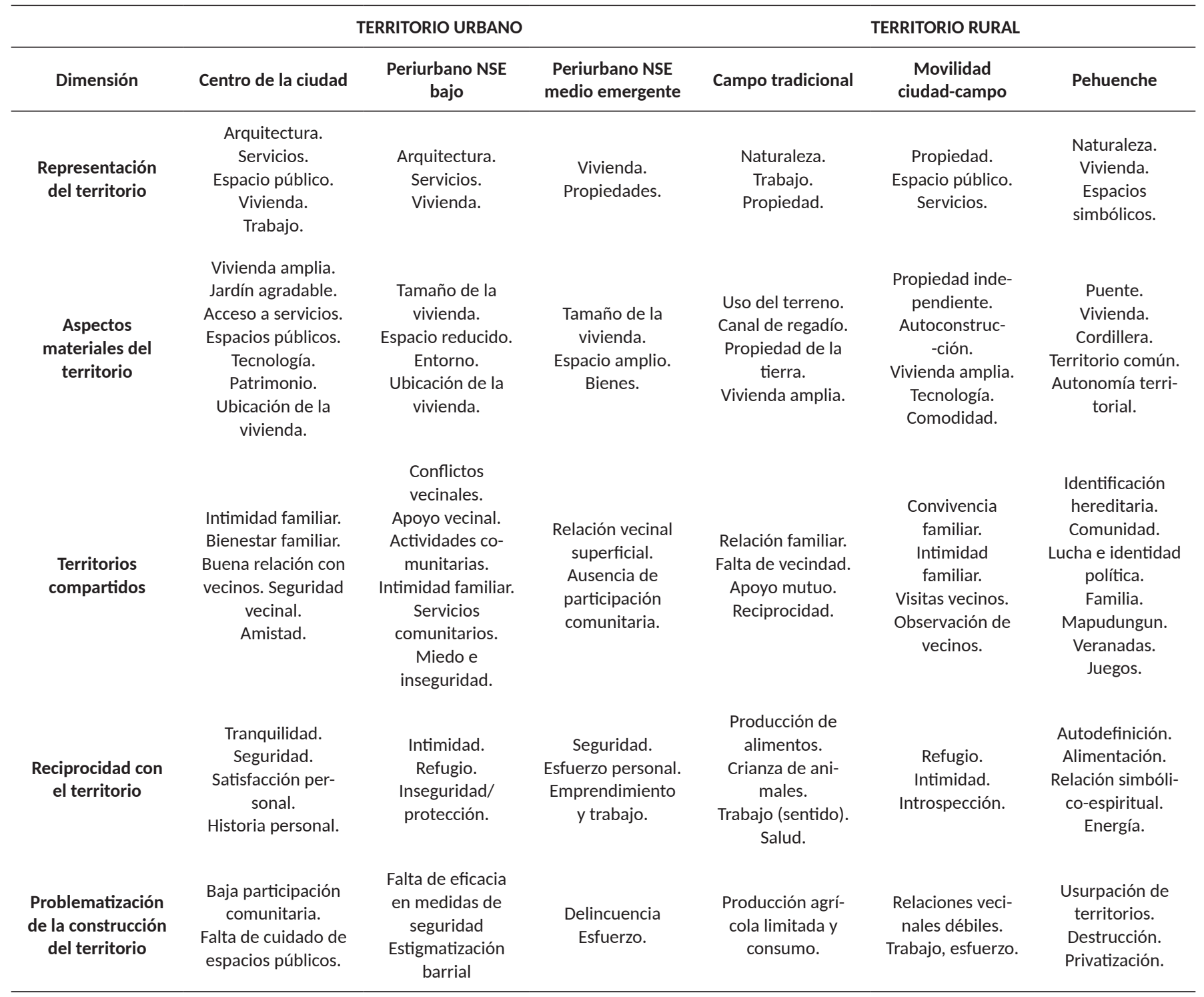

Fuente: elaboración propia.

Los territorios compartidos son definidos a partir de la relación con otros. En el caso del territorio del centro urbano, estas relaciones se dan centralmente en la intimidad y bienestar del espacio familiar, así también se destaca la buena relación con vecinos y la seguridad que brinda este espacio urbano, presentándose en una situación de privilegio debido a la seguridad y el "acceso a todo" y la seguridad. En el caso de la periferia urbana la temática de justificación de las relaciones con otros se centra en la intimidad familiar, el miedo e inseguridad, justificados en relatos en torno al peligro, al daño por parte de sujetos del barrio u otros. En el caso de la Periferia Urbana nivel socioeconómico bajo, se da cuenta de relaciones con otros mediadas por la participación en servicios comunitarios (centros de salud, iglesias, juntas vecinales, etc.) y actividades comunitarias. Mientras que, en el caso de Periferia Urbana nivel socioeconómico medio emergente, se expresa ausencia de participación comunitaria. Otro contraste entre estos dos tipos de periurbanidad son las relaciones vecinales, en el caso NSE bajo, estas se matizan entre conflictos vecinales y el apoyo mutuo vecinal, mientras que el NSE medio emergente, describe relaciones vecinales superficiales, limitados por una dedicación exclusiva al trabajo y la familia. En donde, a pesar de la homogeneidad de la vivienda y el barrio en general, se mantiene una vida privada diferenciada gracias a la propiedad y seguridad de la infraestructura (rejas, alarmas de seguridad, etc.).

En el caso del campo, los territorios compartidos se asocian a la convivencia e intimidad familiar. También 
se describen las relaciones vecinales, en donde, el territorio del campo tradicional es descrito en base a prácticas de apoyo mutuo y reciprocidad, en cambio, el territorio movilidad ciudad-campo, se caracteriza por falta de vecindad, asumiendo una actitud contemplativa de la naturaleza, escasa relaciones y/o visitas esporádicas con los vecinos. Sin embargo, se mantiene el proyecto íntimo del territorio adquirido como propiedad privada para este fin. Finalmente, en el caso del territorio pehuenche las relaciones con otros en el territorio se centran en la comunidad, los usos del idioma mapudungun, las relaciones con la familia extendida y prácticas de gran relevancia comunitaria, por ejemplo, juegos tradicionales y el cuidado de animales mediante una práctica cultural denominada veranadas. Por otro lado, se atribuye a las relaciones con otros una vinculación con la identificación hereditaria, lucha e identidad política por el territorio.

La reciprocidad del territorio se da a partir del vínculo entre el sujeto, su entorno y la influencia que el lugar habitado ejerce sobre este. En el caso del territorio del centro urbano, esta relación se atribuye a la tranquilidad, seguridad, satisfacción personal y una vinculación de lugares con hitos de su historia personal. En el caso de la periferia urbana, la reciprocidad se justifica en una emocionalidad asociada a la intimidad y el refugio, es decir, en un binario entre la inseguridad en el espacio público y la protección en el espacio privado. En el caso de la periferia urbana nivel socioeconómico medio emergente el territorio se relaciona con el esfuerzo personal, el emprendimiento y el trabajo. En el caso del campo, la reciprocidad con el territorio se expresa en prácticas cotidianas, en el caso de campo tradicional se vincula a la producción de alimentos, la crianza de animales y el trabajo como otorgador de sentido existencial y de bienestar en la salud. Mientras que, en el caso movilidad ciudad-campo, la relación de reciprocidad se expresa en una emocionalidad asociada a la introspección debido a las posibilidades de refugio e intimidad en el territorio como lugar personal. Finalmente, en el caso del territorio pehuenche, la reciprocidad con el territorio es central, ya que se le atribuyen aspectos identitarios en una relación material y simbólica orientada a su alimentación y energía espiritual.

Por último, respecto a la problematización en la construcción del territorio, en la cual se describen posibles relaciones de poder e influencia de otros, se observa que, para el caso centro urbano, se problematiza una baja participación comunitaria, así como también una falta de cuidado de espacios públicos (áreas verdes y arquitectura patrimonial). En el caso de la periferia urbana, se problematiza la estigmatización barrial (nivel socioeconómico bajo), la delincuencia y el aislamiento familiar como respuesta a la falta de eficacia en medidas de seguridad en el barrio. También se destacan la falta de acceso a una vivienda digna. En el caso del campo, se cuestiona la producción agrícola industrial versus la producción limitada y autoconsumo del campo tradicional fruto del trabajo. En el caso de movilidad ciudad-campo se problematizan las relaciones vecinales débiles a propósito del mantenimiento del desarrollo vital de sus integrantes en la ciudad (trabajo, estudio, etc.). Finalmente, en el caso del territorio pehuenche, se problematiza la usurpación histórica de territorios, la privatización de territorios comunitarios y la destrucción de la naturaleza por parte de la empresas forestales, hidroeléctricas y legitimadas por el Estado de Chile.

\section{Consideraciones finales}

Comprender la construcción subjetiva del territorio, contribuye a comprender distintas formas de referir el territorio constituido en base a aspectos materiales y subjetivos. En este caso se han graficado en cuatro grandes ámbitos: 1) las características conceptuales y materiales del territorio, 2) las relaciones comunitarias en el territorio, 3) el vínculo entre el sujeto y su espacio habitado y finalmente 4) la problematización de las relaciones de poder e influencia de otros en las formas de apropiación de los espacios habitados.

A partir de lo anterior, podemos comprender las relaciones que las personas mantienen con sus espacios inmediatos, como relaciones en las cuales los individuos conceptualizan y significan su espacio en función de las experiencias internalizadas como parte de su propia realidad, tal como lo conceptualizan Vidal y Pol (2005) y Vidal et al., (2004). En este sentido, las configuraciones espaciales de los lugares se viven y se les da un significado en función de las relaciones que tenemos con los otros y del tipo de vínculo que se constituya en estas relaciones. Además, la representación del territorio tiene directa relación con la experiencia espacial que se tenga con la materialidad de este, es decir, con la funcionalidad que el espacio brinde a la experiencia del lugar, favoreciendo la identidad relacionada al entorno físico (Berroeta et al., 2015).

En este sentido, la representación del territorio, sin desconocer la agencia de lo material, no se puede comprender sólo desde los servicios, la arquitectura o la vivienda, sino que estos elementos constitutivos del espacio adquieren significación en la medida en que se 
comprenda el tipo de vínculo afectivo y racional que los individuos adquieran con el lugar (Hidalgo, 1998). Por otro lado, la cualificación del territorio guarda relación con la identidad comunitaria y las distintas formas de participación en los espacios habitados, promueve que las personas realicen conexiones entre sus experiencias individuales y comunitarias en sus barrios, evidenciando una relación entre el sentimiento de comunidad y la caracterización del mundo material. Desde esta perspectiva, y en concordancia con la noción de sentido de comunidad (Montero, 2004), las relaciones con los lugares están definidos por relaciones con otros, las cuales dan sentido a la materialidad, produciendo significados asociados a la convivencia, la seguridad, la intimidad familiar, buena vecindad, etc. En este sentido, la materialidad del territorio no define las relaciones persona- entorno por sí solas, sino que contribuye a moderar las relaciones comunitarias y la apropiación del espacio en una relación de interdependencia, la cual permite complejizarlas y llevarlas a una esfera de problematización de los problemas experimentados en la interacción. Este aspecto de la participación forma parte de un proceso gradual (en la interacción constante), permite la visibilización, la disputa y ejercicio del poder y con esto, la posibilidad de tomar decisiones, visibilizando situaciones de desigualdad o incomodidad como la falta de cuidado del espacio público y/o la estigmatización barrial en el caso urbano y/o la usurpación de territorios en el caso rural.

Estos antecedentes sirven para seguir problematizando las múltiples relaciones y significados en torno a un territorio, los cuales pueden ser comprendidos desde la relación entre la materialidad, los discursos y la experiencia de quienes están inmersos en él en lo cotidiano. De esta manera, los territorios, diferenciados por sus características geográficas, tienen múltiples diferencias y sus discursos promueven distintas subjetivaciones al interior de sus mismos campos, lo que posibilita hablar de una construcción subjetiva del territorio como una red de relaciones que nos presenta el desafío de investigar más allá de la materialidad y los discursos, es decir, considerar la interacción de estos ámbitos en la cotidianidad, lo cual puede ser una contribución para el dialogo ya iniciado en las ciencias sociales interesadas en la comprensión de la relación entre las personas y los entornos naturales, apropiados o imaginados. Esto, tal vez, puede ser facilitado por la metodología expuesta en este artículo, aprovechando su multidimensionalidad y la riqueza de los datos que puede producir.

\section{Referencias}

Altman, I., \& Low, S. M. (1992). Place Attachment. Nueva York: Plenum Press.

Amérigo, M. (1990). Satisfacción residencial. Una aproximación psicosocial a los estudios de calidad de vida. Madrid: Universidad Complutense.

Ander-Egg, E. (1980). Noción de comunidad. En Ander-Egg, Metodología y práctica del desarrollo de la comunidad (pp. 43-46) Tarragona: Universitaria Europea.

Ávila, M. G. (2002). Aspectos éticos de la investigación cualitativa. Revista Iberoamericana de Educación, 29, 85-103. Extraído de https://rieoei.org/RIE/article/view/952

Azócar, G., Henríquez, C., Valenzuela, C., \& Romero, H. (2008) Tendencias sociodemográficas y segregación socioespacial en Los Ángeles Chile. Revista de Geografia Norte Grande, 128(41), 103-128. doi: 10.4067/S0718-34022008000300006

Bernardo, F., \& Palma-Olivera, J. (2016). Urban neighbourhoods and intergroup relations: The importance of place identity. Journal of Environmental Psychology, 45, 239-251. doi: 10.1016/j. jenvp.2016.01.010

Berroeta, H., \& Zambrano, A. (comp.) (2012). Teoría y Práctica de la Acción Comunitaria. Santiago de Chile: RIL Editores.

Berroeta, H., Ramoneda, A., Rodríguez, V., Di Masso, A., \& Vidal, T. (2015). Apego al lugar, identidad de lugar, sentido de comunidad y participación cívica en personas desplazadas de la ciudad de Chaitén. Magallania, 43(3), 51-63. doi: 10.4067/ S0718-22442015000300005

Brown, G., Raymond, C., \& Corcoran, J. (2015). Mapping and measuring place attachment. Applied Geography, 57, 42-53. doi: 10.1016/j. apgeog.2014.12.011

Casakin, H., Hernández, B., \& Ruiz, B. (2015). Place Attachment and place identity in Israeli cities: The influence of the city size. Cities, 42, 224-230. doi: 10.1016/j.cities.2014.07.007

Conde, F. (2009). Análisis sociológico del sistema de discursos (n. 43). Madrid: CIS Colección Cuadernos Metodológicos.

Davis, A. (1982). Participation in community intervencion design. American Journal of Community Psychology, 10(4), 429- 446. doi: 10.1007/BF00893981

Debord, G. (2006). Introduction to a critique of urban geography. En K. Knabb (Ed.), Situationist international anthology (pp. 23-27). Berkeley, California: Bureau of Public Secrets.

De la Torre, C (2001). Las identidades: una mirada desde la psicología. La Habana: Centro de investigación y desarrollo de la cultura Cubana Juan Marinello.

Devine-Wright, P., \& Clayton, S. (2010). Introduction to the special issue: Place, identity and environmental behavior. Journal of Environmental Psychology, 21(3), 273-281. doi: 10.1016/S0272-4944(10)00078-2

Farmer, J., Currie, M., Kenny, A., \& Munoz, S. (2015). An exploration of the longer-term impacts of community participation in rural health services design. Social Science \& Medicine, 141, 64-71. doi: 10.1016/j.socscimed.2015.07.021

Fernández, R. (2007). Los lugares de la memoria; del golpe y la dictadura militar en Chile: un análisis autoetnográfico de la marcha del 11 de septiembre. Cuadernos de Neuropsicología, 1(2), 150-164. Extraído de http://pepsic.bvsalud.org/scielo.php?script=sci_arttext\&pid=S0718-41232007000200006\&lng=pt\&tlng=es 
Ferullo, A. (2006). El Triángulo de las tres "P”: psicología, Participación y poder. Buenos Aires: Paidós.

Gergen, K. (2007). Construccionismo social. Aportes para el debate y la práctica. Colombia: Unidades.

Giner, S., Lamo, E., \& Torres, C. (1998). Diccionario de sociología. Madrid: Alianza Editorial.

Giuliani, M. V. (2003). Theory of attachment and place attachment Psychological theories for environmental issues. Aldershot: Ashgate.

Giuliani, M. V., \& Feldman, R. (1993). Place attachment in a developmental and cultural context. Journal of Environmental Psychology, 13(3), 267-274. doi: 10.1016/S0272-4944(05)80179-3

Harris, P., Brown, B., \& Werner, C. (1996). Privacy regulation and place attachment: predicting attachments to a student family housing facility. Journal of Environmental Psychology, 16, 287-301. doi: 10.1006/ jevp.1996.0025

Hernández, B., Hidalgo, C., Salazar-Laplace, E., \& Hess, S. (2007). Place attachment and place identity in natives and non-natives. Journal of Environmental Psychology, 27, 310-319. doi: 10.1016/j. jenvp.2007.06.003

Hernández, R., Fernández, C. \& Baptista, P. (2006). Metodología de la investigación. México D.F.: McGraw-Hill Interamericana.

Hidalgo, M. C. (1998). Apego al lugar: ámbitos, dimensiones y estilos. España: Universidad de La Laguna.

Hidalgo, M. C., \& Hernández, B. (2001). Place attachment: conceptual and empirical questions. Journal of Environmental Psychology, 21(3), 273-281. doi: 10.1006/jevp.2001.0221

Holton, M. (2015). Adapting relationships with place: Investigating the evolving place attachment and 'sense of place' of UK higher education students during a period of intense transition. Geoforum, 59, 21-29. doi: 10.1016/j.geoforum.2014.11.017

Hombrados-Medieta, I. (2010). Calidad de vida y sentido de comunidad en la ciudad. Revista Uciencia, 3, 38-41. Extraído de http://www. uciencia.uma.es/Revista-Uciencia/Ellas-investigan

Hombrados-Medieta, I., Gomez-Jacinto, L., \& Domínguez-Fuentes, J. M. (2009). The impact of immigrants on the sense of community. Journal of Community psychology, 37(6), 671-683. doi: 10.1002/ jcop. 20323

Iñiguez, L. (2005) Nuevos debates, nuevas ideas y nuevas prácticas en la psicología social de la era "post-construccionista". Athenea Digital, 8, 1-7. doi: 10.5565/rev/athenead/v1n8.235

Jorgensen, B., \& Stedman, R. (2001). Sense of place as an attitude: lakeshore owners attitudes toward their properties. Journal of Environmental Psychology, 21, 233-248. doi: 10.1006/ jevp.2001.0226

Joutard, F. (1999). Esas voces que nos llegan del pasado. Buenos Aires: FCE

Kamalipour, H., Yeganeh, A., \& Alalhesabi, M. (2012). Predictors of place attachment in urban residential environments: a residential complex case study. Procedia - Social and Behavioral Sciences, 35, 459-467. doi: 10.1016/j.sbspro.2012.02.111

Korosec-Serfaty, P. (1976). Appropeiation of space. Proceedings of the Strasbourg conference. IAPC-3. Strasbourg-Lovaine La Neuve: CIACO.

Krause, M. (2001). Hacia una redefinición del concepto de comunidad - cuatro ejes para un análisis crítico y una propuesta. Revista de Psicología, 10(2) 44-60. doi: 10.5354/0719-0581.2001.18572
Krause, M. (2007). Hacia una redefinición del concepto de comunidad: cuatro ejes para un análisis crítico y una propuesta. En J. Alfaro \& $\mathrm{H}$ Berroeta (Eds.), Trayectoria de la psicología comunitaria en Chile: prácticas y conceptos (pp. 245-258). Valparaíso, Chile: Universidad de Valparaíso.

Krause, M., \& Jaramillo, A. (1998) Intervenciones psicológico-comunitarias en Santiago de Chile. Santiago: Pontificia Universidad Católica de Chile.

Lewicka, M. (2010). Place attachment: How far have we come in the last 40 years? Journal of Environmental Psychology, 31, 207-230. doi: 10.1016/j.jenvp.2010.10.001

Mardones, R. E. (2013). Notas para un dialogo entre la psicología ambiental comunitaria y la geografía crítica. En Anales de la sociedad chilena de ciencias geográficas (pp. 209-216). Santiago: SOCHIGEO.

Mardones, R. E. (2014). Espacialidad y construcción de lugar: el caso del holograma espacial de la violencia de género. Psicoperspectivas, 13(2), 79-87. doi: 10.5027/ psicoperspectivas-Vol13-Issue2-fulltext-418

Mardones, R. E. (2016). Discurso del territorio enunciado por las ciencias sociales en las publicaciones científicas de revistas latinoamericanas. Prisma Social. Revista de Investigación Social, 16, 556596. Extraído de http://revistaprismasocial.es/article/view/1266

Mardones, R. E., Ulloa, J. B. M., \& Salas, G. (2018). Usos del diseño metodológico cualitativo en artículos de acceso abierto de alto impacto en ciencias sociales. Forum Qualitative Sozialforschung/ Forum: Qualitative Social Research, 19(1). doi: 10.17169/ fqs-19.1.2656

Martínez, V. (2006). El enfoque comunitario. El desafío de incorporar a la comunidad en las intervenciones sociales (Tesis de Magíster). Santiago de Chile: Universidad de Chile.

Mc Millan, D., \& Chavis, D. (1986). Sense of community: a definition and theory. Journal of Community Psychology. 14(1), 6-23. doi: 10.1002/1520-6629(198601)14:1\%3C6::AIDJCOP2290140103\%3E3.0.CO;2-I

Moghisi, R., Mokhtari, S., \& Heidari, A. (2015). Place attachment in university students. Case study: Shiraz University. Procedia Social and Behavioral Sciences, 170, 187-196. doi: 10.1016/j. sbspro.2015.01.028

Montenegro, M., Rodriguez, A., \& Pujol, J. (2014). La Psicología Social Comunitaria ante los cambios en la sociedad contemporánea: de la reificación de lo común a la articulación de las diferencias. Psicoperspectivas, 13(2), 32-43. doi: 10.5027/ psicoperspectivas-Vol13-Issue2-fulltext-352

Montero, M. (2004). Introducción a la Psicología comunitaria. Desarrollo, conceptos y procesos. Buenos Aires: Paidós.

Montesinos, A. (2013). Práctica y representación del territorio como ejercicio de enunciación. Algunas propuestas personales. URBS Revista de Estudios Urbanos y Ciencias Sociales. 4(1). 301-311. Extraído de http://www2.ual.es/urbs/index.php/urbs/article/view/ ruiz_montesinos/164

Pantoja, S. R. (2011). Escuela e identidad: un desafío docente para la cohesión social. POLIS, 10(30), 447-476. doi: 10.4067/ S0718-65682011000300021

Pellicer, I., Nubiola, C., \& De Lacour, R. (2013). Derivas: arte, ciudad y sociedad. URBS. Revista de Estudios Urbanos y Ciencias Sociales, 4(1). 9-20. Extraído de http://www2.ual.es/urbs/index.php/urbs/ article/view/pellicer_nubiola_lacour/171 
Pérez, C. (2009). Sobre la condición social de la Psicología. (2º Edición). Santiago: LOM.

Pick, S., Sirkin, J., Osorio, P., Xocolotzin, U., \& Givaudan, M. (2007). Escala para medir agencia personal y empoderamiento (ESAGE). Revista Interamericana de Psicología, 41(2), 295-304. Extraído de http://pepsic.bvsalud.org/scielo.php?script=sci_arttext\&pid=S0034-96902007000300004\&lng=pt\&tIng=es

Pol, E. (1996). La apropiación del espacio. En L. Iñiguez \& E. Pol (Eds.), Cognición, representación y apropiación del espacio (Vol. 9). Barcelona: Publicacions Universitat de Barcelona.

Prezza, M., Zampatti, E., Pacilli, M., \& Paoliello, A. (2008). Territorial sense of community, ethnic prejudice and political orientation. Journal of Community and Applied Social Psychology, 18, 315-338. doi: $10.1002 /$ casp.927

Proshansky, H. M. (1978). The city and self-identity. Environment and Behavior, 10(2), 147-169. doi: 10.1177/0013916578102002

Proshansky, H. M., Fabian, A. K., \& Kaminoff, R. (1983). Place-identity: physical world socialization of the self. Journal of Environmental Psychology, 3(1), 57-83. doi: 10.1016/S0272-4944(83)80021-8

Qinjiu, S., \& Maliki, N. (2013). Place attachment and place identity: Undergraduate students place bonding on campus. Procedia - Social and Behavioral Sciences, 91, 632-639. doi: 10.1016/j. sbspro.2013.08.463

Sánchez, A. (1996). Psicología Comunitaria. Bases conceptuales y métodos de intervención. Barcelona: EUB

Sánchez, A. (2013). ¿Es posible el empoderamiento en tiempos de crisis? Repensando el desarrollo humano en el nuevo siglo. Universitas Psychologica, 12(1), 285-300. Extraído de http://www.scielo.org.co/ scielo.php?script=sci_arttext\&pid=S1657-92672013000100025\&ln$\mathrm{g}=$ en\&tlng=es.

Scannell, L., \& Gifford, R. (2010). Defining place attachment: A tripartite organizing framework. Journal of Environmental Psychology, 30, 1-10. doi: 10.1016/j.jenvp.2009.09.006

Smith, N., Padgett, D., Choy-Brown, M., \& Henwood, B. (2015). Rebuilding lives and identities: the role of place in recovery among persons with complex needs. Health \& Place, 33, 109-117. doi: 10.1016/j.healthplace.2015.03.002

Subercaseaux, B., Rojo, G., Oyarzún, K., Ruiz, C., Martínez, J. L., Wallace, D.; ... Baeza, A. (2002), Identidades y sujetos. Para una discusión latinoamericana. Santiago de Chile: Ediciones Facultad de Filosofía y Humanidades, Universidad de Chile.
Taylor, S. J., \& Bogdan, R. (1987). Introducción a los métodos cualitativos de investigación: la búsqueda de significados. Barcelona: Paidós.

Tuan, Y. (2001). Space and place: The perspective of experience. Minneapolis, USA: University of Minnesota Press.

Turner, J. C. (1990). Redescubrir el grupo social. Una teoría de la categorización del yo. Madrid: Morata

Twigger-Ross, C., \& Uzzell, D. (1996). Place and identity processes. Journal of Environmental Psychology, 16(3), 205-220. doi: 10.1006/ jevp.1996.0017

Ucar, X. (2012). La comunidad como elección. En H. Berroeta \& A. Zambrano (Eds.), Teoría y Práctica de la Acción Comunitaria. Aportes desde la psicología comunitaria (pp. 37-73). Santiago de Chile: RIL Editores.

Ujang, N., \& Zakariya, K. (2015). Place attachment and the value of place in the life of users. Procedia - Social and Behavioral Sciences, 168(9), 373-380. doi: 10.1016/j.sbspro.2014.10.243

Valles, M. (1999). Técnicas cualitativas de investigación social. Reflexión metodológica e investigación social. Madrid: Editorial síntesis.

Vidal, T., Berroeta, H., Di Masso, A., Valera, S., \& Peró, M. (2013) Apego al lugar, identidad de lugar, sentido de comunidad y participación en un contexto de renovación urbana. Estudios de Psicología, 34(23), 275-286. doi: 10.1174/021093913808295172

Vidal, T., \& Pol, E. (2005). La apropiación del espacio: una propuesta teórica para comprender la vinculación entre las personas y los lugares. Anuario de Psicología. 36(3), 281-297. Extraído de http:// www.raco.cat/index.php/AnuarioPsicologia/article/view/61819

Vidal, T., Pol, E., Guàrdia, J., \& Peró, M. (2004). Un modelo de apropiación del espacio mediante ecuaciones estructurales. Medio Ambiente y Comportamiento Humano, 5(1 y 2), 27-52. Extraído de https://mach.webs.ull.es/PDFS/Vol5_1y2/VOL_5_1y2_b.pdf

Vidal, T., Valera, S., \& Peró, M. (2010). Place attachment, place identity and residential mobility in undergraduate students. Psyecology, 1(3), 54-66. doi: 10.1174/217119710792774799

Villela, P. M. (1976). Espace Appropie, espace Appropiant. Trabajo presentado en L'appropiation de l'espace, Strasbourg, Francia.

Zambrano, A., Bustamante, G., \& Garcia, M. (2009). Trayectorias organizacionales y empoderamiento comunitario: un análisis de interfaz en dos localidades de la región de la Araucanía. Psykhe, 18(2), 65-78. doi: 10.4067/S0718-22282009000200005

Rodolfo E. Mardones, Dr. (c) en Ciencias Sociales por la Universidad de la Frontera, Chile. Es Profesor de la Universidad Austral de Chile (UACh). Dirección para correspondencia: Escuela de Psicología, Facultad de Medicina, Universidad Austral de Chile, Campus Isla Teja, Valdivia, Chile. Teléfono: +56632276475 . Correo electrónico: rodolfo.mardones@uach.cl

Jorge Ulloa, Dr. (c) en Ciencias Sociales por la Universidad de la Frontera, Chile. Es Profesor de la Universidad Santo Tomás, Chile (UST). Correo electrónico: jorge.ulloa@ufrontera.cl 